\title{
FACTORES RELACIONADOS CON LAS DIFERENTES ESTRATEGIAS DE AFRONTAMIENTO AL CÁNCER DE MAMA EN PACIENTES DE RECIÉN DIAGNÓSTICO
}

\author{
FACTORS RELATED WITH DIFFERENT STRATEGIES TO COPE BREAST CANCER IN \\ RECENT DIAGNOSIS PATIENTS
}

María Antonieta De Haro-Rodríguez', Lilia Susana Gallardo-Vidal², Martha Leticia Martínez-Martínez², Nicolás Camacho-Calderón ${ }^{3}$ Jorge Velázquez-Tlapanco 4 y Eduardo Paredes Hernández ${ }^{5}$

1 Unidad de Medicina Familiar 16, Instituto Mexicano de Seguro Social, Querétaro, México.

2 Unidad de Medicina Familiar No 13 Instituto Mexicano de Seguro Social, Querétaro, México.

${ }^{3}$ Facultad de Medicina Universidad Autónoma de Querétaro, México.

${ }^{4}$ Facultad de Medicina de la Universidad Autónoma de Querétaro, México.

${ }^{5}$ Hospital General Regional 1, Instituto Mexicano de Seguro Social, Querétaro, México.

Resumen

Objetivo: Identificar los factores relacionados con las diferentes estrategias de afrontamiento al cáncer de mama en pacientes con reciente diagnóstico. Método: Estudio descriptivo trasversal, de correlación en pacientes con cáncer de mama de la clínica de mama del Hospital General Regional 1 y Unidad Médica de Atención Ambulatoria, del Instituto Mexicano del Seguro Social, Querétaro, México, en el 2011. Se estudiaron 98 pacientes con el diagnóstico referido, entre seis y doce meses de evolución, de cualquier edad. Se estudiaron variables socio-demográficas, tipo de familia, tratamiento recibido, la capacidad y estrategias de afrontamiento utilizadas, medidas con el instrumento "Inventario de Estrategias de Afrontamiento" modificado y adaptado al castellano por Cano et al. en el año 2007, replicado en México en el 2010 por Nava et al. Se respetaron las consideraciones éticas. Se analizó con estadística descriptiva, Rho de Spearman, Pearson y con la $X^{2}$. Resultados: El sentimiento que con mayor frecuencia se presentó al recibir el diagnóstico fue el temor $(28,6 \%)$, la estrategia de afrontamiento más usada fue

\section{Abstract}

Objetive: To identify factors relating to the strategies for coping with breast cancer recently diagnosed patients. Methods: cross-sectional design, descriptive study of correlation among patients with breast cancer at the Clinica de Mama of the Hospital General Regional $N^{\circ} 1\left(\mathrm{HGR} \mathrm{N}^{\circ} 1\right)$ and Unidad Medica de Atencion Ambulatoria (UMAA), Instituto Mexicano del Seguro Social, Queretaro, Mexico in 2011. 98 patients of all ages with this diagnosis were studied with a background of diagnosis of between six to twelve months. Sociodemographic variables were studied (age, schooling, occupation, marital status), type of family, type of treatment received, and capability for coping strategies by them with "Inventory of strategies of coping", modified and adapted in Spain by Cano et al. (2007) and reproduced in Mexico by Nava et al.(2010). In all procedures were applied with ethical considerations. An analysis was carried out using descriptive statistics, Spearman's Rho, Pearson's $r$ and $X^{2}$. Results: The predominant emotion upon hearing the diagnosis was fear $(28,6 \%)$, the most coping strategy was

\section{Correspondencia:}


la resolución de problemas. Los factores que se vieron estrechamente relacionados con el afrontamiento fueron la edad, la escolaridad, el tipo de familia y tratamiento utilizado. Conclusiones: El tipo de familia, de tratamiento recibido la edad y la escolaridad se encuentran estrechamente relacionados con el estilo de estrategias de afrontamiento que presentan las pacientes con cáncer de mama.

Palabras clave: Cáncer de mama, afrontamiento, reciente diagnóstico. problem solving. The related factors with the ability to cope were age, schooling, type of treatment received. Conclusions: Type of family, treatment received, age and schooling are strongly related tocoping strategies among patients with recent diagnosed breast cancer.

Keywords: Breast cancer, coping, recent diagnosis.

\section{INTRODUCCIÓN}

El diagnóstico de cáncer es considerado un evento estresante ${ }^{(1,2)}$. En particular el cáncer de mama, ya que es visto por la población como una enfermedad de pronóstico oscuro ${ }^{(3)}$, mutilante, $\operatorname{costoso}^{(4,5)}$, que daña a nivel personal ${ }^{(6-8)}$, familiar ${ }^{(8,9),}$ de pareja, laboral y social, al ser considerado como una amenaza persistente ${ }^{(10)}$; que causa efectos en la mayoría de las ocasiones negativos en los aspectos biológico, psicológico y social ${ }^{(11-14)}$.

El proceso, que acompaña al notificar dicho diagnóstico a la afectada, requiere de mecanismos de reconstitución psíquica que permitan organizar una respuesta a los retos que crea este padecimiento ${ }^{(15,17)}$. Para que esto se pueda dar se requieren mecanismos adaptativos Ilamados estrategias de afrontamiento $^{(18)}$ que permitan la capacidad estabilizadora ante una situación de estrés ${ }^{(18-21)}$. Dentro de los mecanismos, se encuentran los cognitivos, afectivos y conductuales, y de las estrategias de afrontamiento, se tienen aquellas adaptativas como la resolución de problemas, reestructuración cognitiva, apoyo social, expresión emocional y las no adaptativas como evitación de problemas, pensamiento desiderativo, retirada social y la autocrítica ${ }^{(19)}$. Existen otros factores que intervienen en la manera de afrontar dicho evento, entre los que se tiene, a la patología y el tipo de tratamiento otorgado ${ }^{(8,14,15)}$, a las características propias del paciente (edad, estado civil, escolaridad, ocupación, nivel socioeconómico) ${ }^{(16)}$, los relacionados con el ambiente del paciente (tipo de familia, apoyo social, relación satisfactoria con su cónyuge, funcionamiento familiar, satisfacción marital) ${ }^{(16-23)}$.

A pesar de esto, la medicina en la actualidad al fraccionarse en diferentes especialidades va perdiendo su visión integradora, y en muchas de las ocasiones la paciente con cáncer de mama es manejada solamente desde el ámbito biológico, por lo que vive de manera personal su duelo, y utiliza sus propios recursos para llevar a cabo el afrontamiento ${ }^{(24,25)}$. Pocos son los países que tienen dentro de sus sistemas de salud un programa psicooncológico bien estructurado que le permita a estas pacientes tener una visión más adaptativa de su enfermedad ${ }^{(26,27)}$. A pesar de que las sociedades han cambiado, las investigaciones relacionadas con los factores psicosociales ${ }^{(28)}$ y familiares ${ }^{(29,30)}$ que influyen en la capacidad de afrontamiento y el incremento de la calidad de vida del paciente y su familia no se han actualizado ${ }^{(21,22)}$.

Por lo que el objetivo del presente estudio es identificar cual es el sentimiento más frecuente que presentan las pacientes con cáncer de mama al recibir el diagnóstico de mama, cuáles son las estrategias de 
afrontamiento más utilizadas y los factores que pueden estar relacionados con dichas estrategias.

\section{MÉTODO}

Estudio descriptivo de correlación en pacientes con diagnóstico de cáncer de mama de seis meses a un año de evolución, del Hospital General Regional № 1(HGR1) y de la Unidad Médica de Atención Ambulatoria (UMAA) de Instituto Mexicano del Seguro Social (IMSS), delegación Querétaro, México, en el año 2011.

Previo consentimiento informado se aplicó un cuestionario auto-administrado para recabar variables sociodemográficas, tipo de tratamiento médico, tipo de familia, el nivel socioeconómico y estrategias de afrontamiento.

Las estrategias de afrontamiento fueron medidas por medio del "Inventario de estrategias de afrontamiento" adaptado al español por Cano García et al.(31), cuya versión original en inglés es de Tobin, HoIrroyd y Widalde, con 72 items quedó en 40 ítems en la versiójn española. Validado en México en la Universidad Autónoma de México (UNAM) por Nava et al. (2010)(32), el cual evalúa constructos de afrontamiento dirigido al problema y a la emoción. Está integrado por ocho estrategias: 1) Resolución de problemas: estrategias cognitivas y conductuales encaminadas a liberar las emociones que acontecen en el proceso de estrés modificando la situación que lo produce, 2) Reestructuración cognitiva: modifican el significado de la situación estresante, 3) Apoyo social: estrategia referida a la búsqueda de apoyo emocional, 4) Expresión emocional: encaminada a liberar las emociones que acontecen en el proceso de estrés, 5) Evitación de problemas: manejo inadecuado centrado en el problema caracterizado por negación y evitación de pensamientos o actos relacionados con el acontecimiento estresante, 6) Pensamiento desiderativo: manejo inadecuado centrado en el problema que refleja el deseo de que la realidad no fuera estresante con la tendencia a fantasear sobre la realidad, 7) Retirada social: manejo inadecuado centrado en la emoción donde la paciente se aísla de amigos, familiares, compañeros y personas significativas, 8) Autocrítica: manejo inadecuado centrado en la emoción basado en la auto-inculpación por la ocurrencia de una situación ${ }^{(30,31)}$.

Dicho instrumento tiene un alfa de Cronbach superior a 0,7 en cada dimensión. Se mide con una escala de Likert de 0 a 4 puntos: 0) En absoluto, 1) Un poco, 2) Bastante, 3) Mucho, y 4) Totalmente; que define la frecuencia de realización de lo explicado en el enunciado ante la patología y representa lo que sintió, pensó, y pasó al enterarse del diagnóstico y tratamiento que se le ofrecía. También explora la percepción de las pacientes de la autoeficacia percibida por la paciente con la misma escala likert de las ocho estrategias: 1) En absoluto, 2) Un poco, 3) Bastante, 4) Mucho y 5) Totalmente ${ }^{(31)}$. Su evaluación es específica para cada estrategia. El total da un puntaje de cero a veinte por estrategia, entre más alta sea la calificación, es el estilo de afrontamiento que utiliza la paciente. Cabe señalar que la paciente puede utilizar varias estrategias a la vez, ya que no son excluyentes entre sí.

Para identificar cuáles fueron los sentimientos y las estrategias de afrontamiento más frecuentemente utilizadas por las pacientes con cáncer de mama ante el diagnóstico; los resultados fueron analizados por medio de frecuencias, promedios, medidas de tendencia central y de dispersión. Para determinar cómo se relacionan cada una de estas estrategias con los aspectos propios del paciente (edad, estado civil, escolaridad, ocupación, nivel socioeconómico), con el tipo de familia (según estructura, desarrollo, ocupación, demografía) y con el tratamiento propio de la enferme- 
dad (número de procedimientos realizados) se aplicó el coeficiente de correlación de Spearman para la variables cualitativas ordinales, el coeficiente de Pearson para variables cuantitativas y la prueba $X^{2}$ para las variables cualitativas nominales con un nivel de confianza del 95\%.

\section{RESULTADOS}

De 98 pacientes, el promedio de la edad fue de $57 \pm 12$ años (29 a 95) con predominio de las casadas $72,4 \%$, con escolaridad primaria $50 \%$, ama de casa $69.4 \%$ y con un nivel socioeconómico obrero $42,9 \%$ (tabla 1 ).

En relación con el tipo de familia, prevaleció la nuclear 59,2\%, tradicional 53,1\%, obrera $84,7 \%$ y urbana $76,5 \%$ (tabla 2 ).

El $86,7 \%$ de las mujeres recibieron cirugía combinada y ninguna tratamiento conservador; de las principales combinaciones que presentaron, predominó la cirugía mas quimioterapia parenteral $36,7 \%$ y la cirugía combinada con radioterapia y quimioterapia parenteral $18,4 \%$ (tabla 3 ).

\section{Tabla 1. Características sociodemográficas en pacientes con cáncer de mama de reciente diagnóstico}

\begin{tabular}{lcc}
\hline Características sociodemográficas & Porcentaje & IC 95\% \\
\hline Estado civil & & $5,0-17,4$ \\
Soltera & 11,2 & $63,5-81,3$ \\
Casada & 72,4 & $00-6,5$ \\
Divorciada & 3,1 & $6,6-20,0$ \\
Viuda & 13,3 & \\
Escolaridad & & $0,2-8,0$ \\
Analfabeta & 4,1 & $40,1-59,9$ \\
Primaria & 50 & $12,4-28,4$ \\
Secundaria & 20,4 & $4,2-16,2$ \\
Bachillerato & 10,2 & $1,4-10,8$ \\
Universidad & 6,1 & $3,5-14,9$ \\
Técnica & 9,2 & \\
Ocupación & & $60,3-78,5$ \\
Ama de casa & 69,4 & $0,2-8,0$ \\
Obrera & 4,1 & $15,1-31,9$ \\
Empleada & 23,5 & $0,0-6,5$ \\
Profesionista & 3,1 & $0,0-4,8$ \\
Nivel socioeconómico* & & $3,5-14,9$ \\
Alto & 2,0 & $33,1-52,7$ \\
Medio alto & 9,2 & $2,0-12,2$ \\
Medio bajo & 38,8 & \\
Obrero & 42,9 & \\
Marginal & 7,1 & \\
\hline
\end{tabular}

*Instrumento de Graffar.

Fuente: Hoja de recolección de datos de Encuestas aplicada a pacientes de la clínica de mama del Hospital General Regional N¹ y de la Unidad Médica de Atención Ambulatoria del IMSS, Delegación Querétaro. 


\section{Tabla 2. Tipología familiar de las pacientes con cáncer de mama de reciente diagnóstico}

\begin{tabular}{|c|c|c|c|}
\hline \multirow[t]{2}{*}{ Tipo de familia } & \multirow[t]{2}{*}{ Porcentaje } & \multicolumn{2}{|c|}{ Intervalo de confianza al 95\% } \\
\hline & & Superior & Inferior \\
\hline \multicolumn{4}{|l|}{ Según estructura } \\
\hline Nuclear & 59,2 & 49,5 & 68,9 \\
\hline Extensa & 21,4 & 13,3 & 29,5 \\
\hline Extensa compuesta & 12,2 & 5,7 & 18,7 \\
\hline Sola & 7,1 & 3,4 & 11,5 \\
\hline \multicolumn{4}{|l|}{ Según su desarrollo } \\
\hline Tradicional & 53,1 & 43,2 & 63,0 \\
\hline Moderna & 46,1 & 36,2 & 56,0 \\
\hline \multicolumn{4}{|l|}{ Según su ocupación } \\
\hline Profesional & 15,3 & 8,2 & 22,4 \\
\hline Obrera & 84,7 & 77,6 & 91,8 \\
\hline \multicolumn{4}{|l|}{ En base a su demografía } \\
\hline Rural & 1,0 & 0,0 & 3,0 \\
\hline Suburbana & 22,4 & 14,1 & 30,7 \\
\hline Urbana & 76,5 & 68,1 & 84,9 \\
\hline
\end{tabular}

Fuente: Hoja de recolección de datos de encuestas aplicada a pacientes de la clínica de mama del Hospital General Regional Nㅜ1 y de la Unidad Médica de Atención Ambulatoria del IMSS, Delegación Querétaro.

\section{Tabla 3. Tipo de tratamiento recibido por pacientes con cáncer de mama de reciente diagnóstico}

\begin{tabular}{lcc}
\hline Tratamiento & Frecuencia & Porcentaje \\
\hline Cirugía & 6 & 6,1 \\
Radioterapia & 0 & 0 \\
Quimioterapia parenteral & 4 & 4,1 \\
Quimioterapia oral & 3 & 3,1 \\
Cirugía +Quimioterapia parenteral & 36 & 36,7 \\
Cirugía+ Radioterapia +Quimioterapia parenteral & 18 & 18,4 \\
Cirugía+ Radioterapia +Quimioterapia parenteral y oral & 10 & 10,2 \\
Cirugía + Radioterapia & 3 & 3,1 \\
Cirugía + Quimioterapia parenteral y oral & 11 & 11,2 \\
Radioterapia + Quimioterapia parenteral & 1 & 1,0 \\
Cirugía +Quimioterapia oral & 4 & 4,1 \\
Cirugía+ Radioterapia + Quimioterapia oral & 2 & 2,0 \\
\hline
\end{tabular}

Fuente: Hoja de recolección de datos de Encuestas aplicada a pacientes de la clínica de mama del Hospital General Regional N¹ y de la Unidad Médica de Atención Ambulatoria del IMSS, Delegación Querétaro. 
El sentimiento más prevalente al establecer el diagnóstico, fue el temor en el $28,6 \%$, seguido de la tristeza $23,5 \%$. Dentro de los diferentes tipos de estrategias de afrontamiento, las que presentaron más alto puntaje fueron la resolución de problemas $(15 \pm 2,86)$, el apoyo social $(13,1 \pm 3,6)$, y pensamiento desiderativo $(13,0 \pm 3,91)$. Dentro de las que presentaron puntajes más bajos la retirada social $(7,52 \pm 3,64)$ y la autocrítica $(8 \pm 4,96)$ estuvieron presentes (tabla 4).

La edad presentó una correlación negativa con la resolución de problemas, autocrítica, expresión emocional, pensamiento desiderativo, apoyo social, reestructuración cognitiva, y positiva con evitación de problemas y retirada social.
La escolaridad presentó una correlación positiva con la resolución de problemas, autocrítica, expresión emocional, pensamiento desiderativo, apoyo social, reestructuración cognitiva y evitación de problemas a excepción de la retirada social que fue negativa (tabla 5).

Se encontró una relación muy estrecha entre la estructura familiar, la resolución de problemas $(p=0,007)$ y la expresión $(p=0,030)$, así como entre la ocupación familiar y el pensamiento desiderativo $(p=0,020)$. En relación a su demografía se observó una fuerte relación con la expresión emocional, pensamiento desiderativo, reestructuración cognitiva, evitación de problemas y retirada social $(p<0,05)$ (tabla 6).

\section{Tabla 4. Sentimientos $\mathbf{y}$ tipos de estrategias de afrontamiento utilizadas por las pacientes con cáncer de mama de reciente diagnóstico}

\begin{tabular}{|c|c|c|c|c|}
\hline Sentimiento & Porcentaje & \multicolumn{3}{|c|}{ IC 95\% } \\
\hline & & Inferior & \multicolumn{2}{|c|}{ Superior } \\
\hline Temor & 28,6 & 19,7 & \multicolumn{2}{|c|}{37,5} \\
\hline Muerte & 16,3 & 9,0 & \multicolumn{2}{|c|}{23,6} \\
\hline Incertidumbre & 20,4 & 12,4 & \multicolumn{2}{|c|}{28,4} \\
\hline Tristeza & 23,5 & 15,1 & \multicolumn{2}{|c|}{31,9} \\
\hline Enojo & 2,0 & 0,0 & \multicolumn{2}{|c|}{4,8} \\
\hline Tranquilidad & 9,2 & 3,5 & \multicolumn{2}{|c|}{14,9} \\
\hline Tipo de estrategias de afrontamiento* & Media & DS & Min. & Max. \\
\hline Resolución de problemas & 15,5 & 2,86 & 6 & 20 \\
\hline Autocrítica & 8,05 & 4,96 & 0 & 16 \\
\hline Expresión de emociones & 12,42 & 4,04 & 0 & 20 \\
\hline Pensamiento desiderativo & 13,03 & 3,91 & 1 & 20 \\
\hline Apoyo social & 13,4 & 3,64 & 2 & 20 \\
\hline Reestructuración cognitiva & 12,08 & 4,11 & 0 & 20 \\
\hline Evitación de problemas & 9,35 & 4,01 & 0 & 17 \\
\hline Retirada Social & 7,52 & 3,6 & 0 & 13 \\
\hline
\end{tabular}

*Obtenido del "Inventario de Estrategias de Afrontamiento" considerando el valor más alto de 20 puntos por estrategia.

Fuente: Hoja de recolección de datos de Encuestas aplicada a pacientes de la clínica de mama del Hospital General Regional N¹ y de la Unidad Médica de Atención Ambulatoria del IMSS, Delegación Querétaro. 
Tabla 5. Correlación entre los factores sociodemográficos de las pacientes con cáncer de mama de reciente diagnóstico y las diferentes estrategias de afrontamiento utilizadas

\begin{tabular}{|c|c|c|c|c|c|c|c|c|c|c|}
\hline \multirow{3}{*}{ Estrategia } & \multicolumn{10}{|c|}{ Variables sociodemográficas } \\
\hline & \multicolumn{2}{|c|}{ Edad } & \multicolumn{2}{|c|}{ Escolaridad } & \multicolumn{2}{|c|}{ Estado civil } & \multicolumn{2}{|c|}{ Ocupación } & \multicolumn{2}{|c|}{$\begin{array}{l}\text { Nivel socioeco- } \\
\text { nómico }\end{array}$} \\
\hline & $r$ & $\mathrm{p}$ & $r$ & $\mathrm{p}$ & $X^{2}$ & $p$ & $X^{2}$ & $\mathrm{p}$ & $X^{2}$ & $\mathrm{p}$ \\
\hline $\begin{array}{l}\text { Resolución de } \\
\text { problemas }\end{array}$ & $-0,26$ & $0,005^{*}$ & 0,11 & 0,148 & 27 & 0,584 & 26 & 0,628 & 39 & 0,506 \\
\hline Autocrítica & $-0,02$ & 0,424 & 0,04 & 0,367 & 40 & 0,767 & 51 & 0,325 & 80 & 0,084 \\
\hline $\begin{array}{l}\text { Expresión emo- } \\
\text { cional }\end{array}$ & $-0,18$ & $0,037^{*}$ & 0,17 & $0,049 *$ & 40 & 0,848 & 46 & 0,671 & 76 & 0,214 \\
\hline $\begin{array}{l}\text { Pensamiento } \\
\text { desiderativo }\end{array}$ & $-0,29$ & $0,002^{*}$ & 9,16 & 0,059 & 38 & 0,735 & 25 & 0,991 & 59 & 0,509 \\
\hline Apoyo Social & $-0,22$ & $0,014 *$ & 9,21 & $0,019 *$ & 39 & 0,688 & 42 & 0,561 & 60 & 0,466 \\
\hline $\begin{array}{l}\text { Reestructuración } \\
\text { cognitiva }\end{array}$ & $-0,31$ & $0,001 *$ & 9,19 & $0,028^{*}$ & 36 & 0,931 & 38 & 0,907 & 51 & 0,934 \\
\hline $\begin{array}{l}\text { Evitación de } \\
\text { problemas }\end{array}$ & 0,11 & 0,138 & 0,05 & 0,329 & 37 & 0,915 & 45 & 0,690 & 66 & 0,523 \\
\hline Retirada social & 0,18 & $0,039 *$ & $-0,07$ & 1,250 & 24 & 0,964 & 47 & 0,177 & 52 & 0,450 \\
\hline
\end{tabular}

*Pruebas de Spearman, Pearson y $\mathrm{X}^{2}$, con un nivel de confianza del $95 \%$.

Fuente: Hoja de recolección de datos de Encuestas aplicada a pacientes de la clínica de mama del Hospital General Regional Nㅜ1 y de la Unidad Médica de Atención Ambulatoria del IMSS, Delegación Querétaro.

\section{Tabla 6. Correlación entre los factores familiares de las pacientes con cáncer de mama de reciente diagnóstico y las diferentes estrategias de afrontamiento utilizadas}

\begin{tabular}{lccccccccc}
\hline & \multicolumn{1}{c}{ Tipo de familia } \\
\hline \multicolumn{1}{c}{ Estrategia } & \multicolumn{1}{c}{ Estructura } & \multicolumn{2}{c}{ Desarrollo } & \multicolumn{2}{c}{ Ocupación } & \multicolumn{2}{c}{ Demografía } \\
\hline & $\mathrm{X}^{2}$ & $\mathrm{p}$ & $\mathrm{X}^{2}$ & $\mathrm{p}$ & $\mathrm{X}^{2}$ & $\mathrm{p}$ & $\mathrm{X}^{2}$ & $\mathrm{p}$ \\
\cline { 2 - 26 } Resolución de problemas & 52 & $0,007^{*}$ & 7,5 & 0,676 & 13 & 0,212 & 16 & 0,694 \\
Autocrítica & 60 & 0,105 & 10 & 0,856 & 13 & 0,663 & 22 & 0,90 \\
Expresión emocional & 71 & $0,030^{*}$ & 22 & 0,184 & 27 & 0,055 & 83 & $0,000^{*}$ \\
Pensamiento desiderativo & 52 & 0,206 & 16 & 0,354 & 28 & $0,020^{*}$ & 81 & $0,000^{*}$ \\
Apoyo Social & 47 & 0,377 & 18 & 0,232 & 11 & 0,733 & 29 & 0,495 \\
Reestructuración cognitiva & 59 & 0,203 & 17 & 0,428 & 10 & 0,881 & 52 & $0,023^{*}$ \\
Evitación de problemas & 36 & 0,943 & 15 & 0,556 & 16 & 0,458 & 52 & $0,022^{*}$ \\
Retirada social & 33 & 0,700 & 19 & 0,120 & 9,8 & 0,709 & 46 & $0,008^{*}$ \\
\hline
\end{tabular}

*Prueba de $\mathrm{X}^{2}$ con un nivel de confianza del $95 \%$.

Fuente: Hoja de recolección de datos de Encuestas aplicada a pacientes de la clínica de mama del Hospital General Regional $N^{\circ} 1$ y de la Unidad Médica de Atención Ambulatoria del IMSS, Delegación Querétaro. 
Hubo correlación negativa entre el nú- con una mayor asociación en las dimenmero de procedimientos realizados y las siones resolución y evitación de problemas diferentes estrategias de afrontamiento, $p \leq 0,05$ (figura 1).

Figura 1. Relación entre el número de procedimientos realizados a las pacientes con recién diagnóstico de cáncer de mama y las diferentes estrategias de afrontamiento utilizadas.

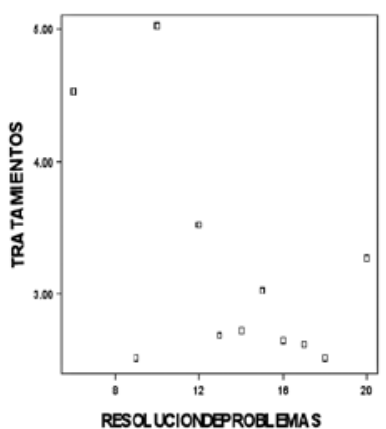

$r=-0,267, p=0,016^{*}$

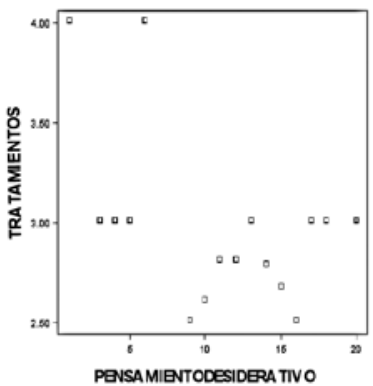

$r=-0,091, p=0,234$

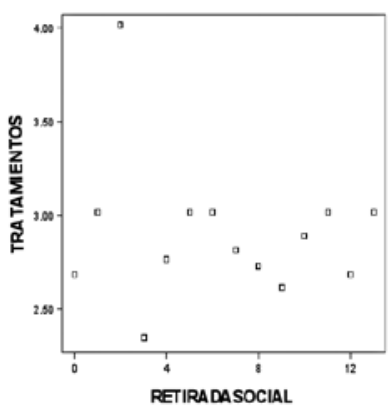

$r=-0,053, p=0,339$

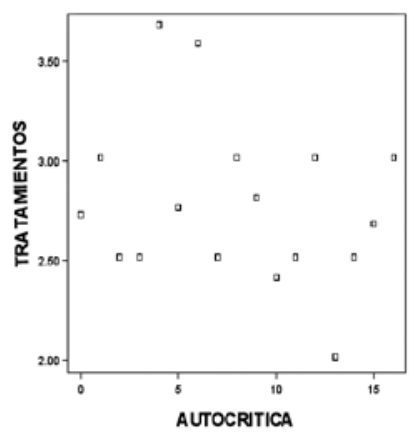

$r=-0,23, p=0,427$

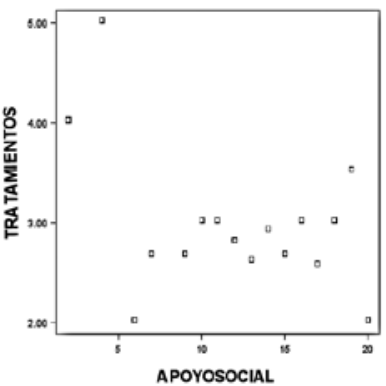

$r=-0,091, p=0,235$

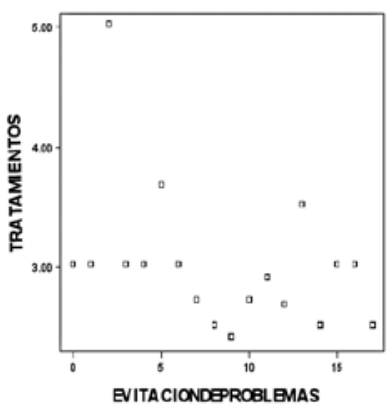

$r=-0,229, p=0,033^{*}$

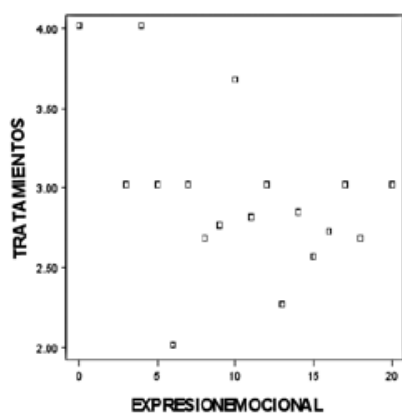

$r=-0,200, p=0,055$

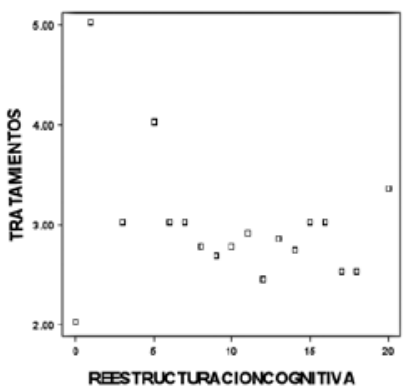

$r=-0,055, p=0,332$

Prueba Rho de Spearman con nivel de confianza del 95\%

Fuente: Hoja de recolección de datos de Encuestas aplicada a pacientes de la clínica de mama del Hospital General Regional $N^{\circ} 1$ y de la Unidad Médica de Atención Ambulatoria del IMSS, Delegación Querétaro. 
La autoeficacia percibida por pacientes, en la mayoría de las estrategias, presentaron una correlación positiva, con una mayor asociación en las dimensiones resolución del problema, expresión emocional, apoyo social y reestructuración cognitiva $\mathrm{p} \leq$ 0, 05 (figura 2).

\section{DISCUSIÓN}

El cáncer de mama en las mujeres representa un problema en su abordaje psicosocial, por la interacción de los factores socioculturales y familiares que le permitirán a la mujer el afrontamiento a su si-

Figura 2. Relación entre la percepción de la auto-eficacia de afrontamiento de las pacientes con recién diagnóstico de cáncer de mama y las diferentes estrategias de afrontamiento utilizadas.

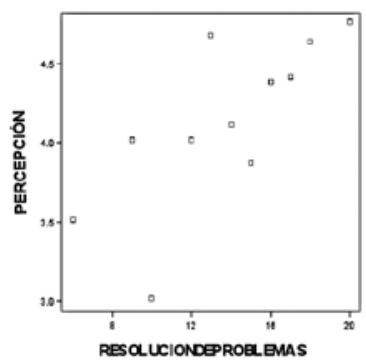

$r=0,393, p=0,001^{*}$

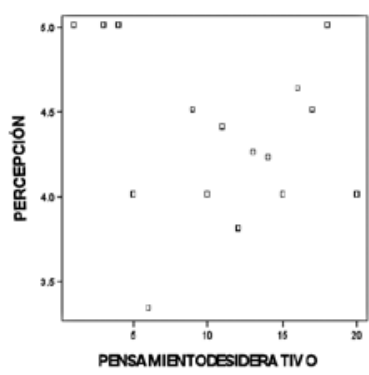

$r=0,188, p=0,067$

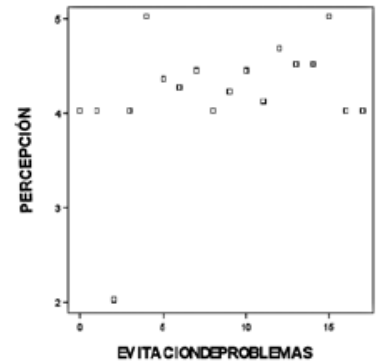

$r=0,059, p=0,319$

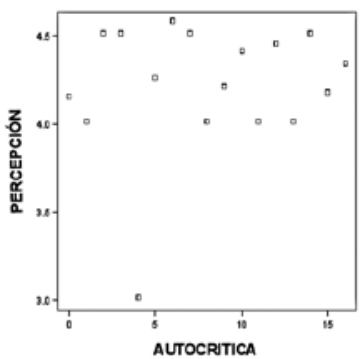

$r=-0,045, p=0,361$

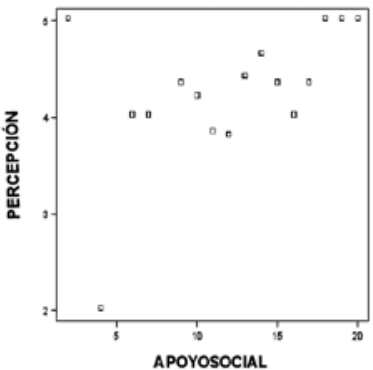

$r=0,282, p=0,011^{*}$

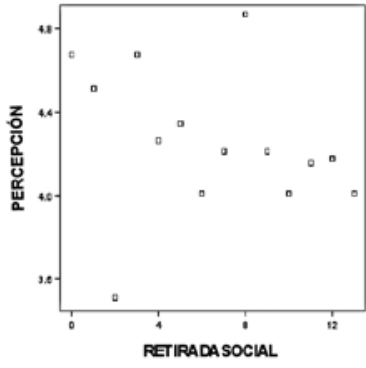

$r=-0,199, p=0,056$

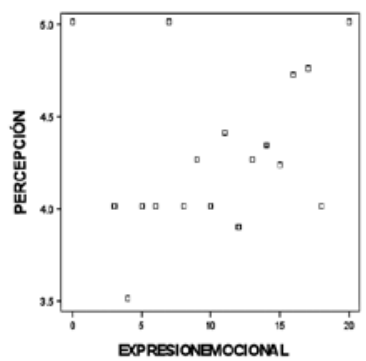

$r=0,229, p=0,034^{*}$

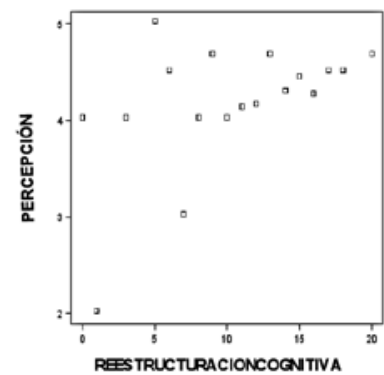

$r=0,251, p=0,022^{*}$

*Prueba Rho de Spearman con nivel de confianza del 95\%

Fuente: Hoja de recolección de datos de Encuestas aplicada a pacientes de la clínica de mama del Hospital General Regional №1 y de la Unidad Médica de Atención Ambulatoria del IMSS, Delegación Querétaro. 
tuación actual de manera más adaptativa, por lo que el abordaje integral en estas pacientes es ineludible.

Respecto a los datos médicos, Ilama la atención, que en la población del presente trabajo se optó en su mayoría por un tratamiento quirúrgico más radical. A ninguna paciente se le realizó tratamiento conservador, diferente a lo encontrado en otros países $^{(12,33)}$, en donde, es un tratamiento muy común, esto puede estar relacionado al estadio en el que se encuentre la paciente en el momento del diagnóstico, lo que haría pensar que en la población mexicana se está diagnosticando en estadios muy avanzados, en donde se requieren tratamientos más radicales, y de forma secundaria también pudiera impactar a nivel psicológico.

De acuerdo a lo encontrado, las pacientes con mayor escolaridad presentan un afrontamiento más adaptativo. Los que alcanzaron significancia estadística dos correspondieron a los centrados en la emoción: expresión emocional y apoyo social y uno centrado en el problema: reestructuración cognitiva; algunos autores consideran que estos estilos de afrontamiento mejoran la calidad de vida(21), por lo cual se pensaría que la población con baja escolaridad puede relacionarse más estrechamente con afrontamientos menos adaptativos.

En la mayoría de los estilos de afrontamiento adaptativos, la edad presentó una correlación negativa, en muchos de estos con una fuerte asociación, diferente a lo encontrado en otros estudios ${ }^{(26)}$ como el de Avis et al. (2013) ${ }^{(34)}$, aunque cabe mencionar que en este último, las mujeres presentaban mayor tendencia a la depresión por los costos generados a la enfermedad ya que muchas de ellas no contaban con algún tipo de seguro. En este estudio, todas las pacientes gozan de servicios de seguridad social, lo que permite en cierta manera disminuir los niveles de estrés y depresión, similar a lo encontrado por Bañuelos-Barrera ${ }^{(6)}$ en donde gran parte de la muestra, que ellos estudiaron, también gozan de seguridad social; aunque cabe mencionar, que en este último estudio la edad presentó una correlación negativa, pero no logró significancia estadística probablemente por un tamaño de muestra pequeño. Un afrontamiento de tipo no adaptativo que presentó significancia estadística fue el pensamiento desiderativo y se correlacionó de forma negativa, lo que hace pensar, que las mujeres más jóvenes, tienden a fantasear más sobre su realidad como un mecanismo de defensa, y las mujeres mayores se apegan más a su realidad.

Tal como la literatura lo muestra ${ }^{(12,33)}$, el tipo de tratamiento médico utilizado afecta directamente en la capacidad de afrontamiento. Se demostró la relación de este con la capacidad de resolución de los problemas, y de acuerdo a esta población, la mayoría recibe terapia combinada (cirugía y quimioterapia) en las que ambas afectan directamente en la calidad de vida de la paciente, por lo que es relevante un diagnóstico oportuno, que además de favorecer en el pronóstico, ayudaría a las pacientes a adaptarse de manera más positiva a su nueva condición. Las estrategias de afrontamiento centradas en el problema, alcanzaron significancia estadística tanto las adaptativas (resolución) como las no adaptativas (evitación),y pareciera que se contradicen, ya que ambas presentaron una correlación negativa, quizá pudiera ser que al recibir mayor combinación de tratamientos, el afrontamiento puede llevarse de forma positiva (resolución) o negativa (evitación), resultados semejantes encontraron Varela y colaboradores en pacientes posterior al tratamiento quirúrgico ${ }^{(33)}$. La expresión emocional se encuentra en el límite para tomarlo como significativo o no, pero se podría pensar que un menor porcentaje de los resultados se explican por el azar. 
Llama la atención que la ocupación y el nivel socioeconómico no presentaron significancia estadística, lo que hace pensar que las mujeres que son diagnosticadas de forma reciente con cáncer de mama, la ocupación no afecta la utilización de los mecanismos adaptativos en el diagnóstico, aunque cabe mencionar, que la mayoría fue ama de casa y esto puede relacionarse con los resultados obtenidos. Era esperado que las pacientes con mayor escolaridad tuvieran mayor capacidad de reestructuración cognitiva, por lo que el grupo de baja escolaridad es vulnerable para los procesos adaptativos. El estado civil no presentó significancia estadística, similar a lo encontrado por Fernández et al.(26). Sería interesante realizar un estudio donde se valoren estos aspectos en pacientes con más tiempo de evolución.

Algo diferente se encontró en la ocupación de los integrantes de la familia de las pacientes, donde sí se observó significancia estadística en el estilo de pensamiento desiderativo, y la ocupación familiar lo que hace pensar que las mujeres con cáncer de mama de reciente diagnóstico pueden fantasear y perderse de su realidad cuando la familia tiene diferentes actividades en el trabajo, de ahí la importancia de la familia como primera red de apoyo durante el proceso de la enfermedad.

Los resultados demuestran que la estructura familiar se relaciona con la capacidad de resolución de los problemas, y la expresión emocional, la demografía en el pensamiento desiderativo, la expresión emocional, la reestructuración cognitiva, la evitación de problemas y la retirada social. Es relevante mencionar las limitaciones de este estudio, ya que no se valoró el funcionamiento familiar ni la satisfacción marital. Díaz y Yarigaño (2010) ${ }^{(34)}$ evaluaron el clima social familiar y el afrontamiento al estrés, en pacientes oncológicos y encontraron una relación muy estrecha con los afrontamientos de tipo adaptativos; Cabrera y Ferraz ${ }^{(29)}$ en ese mismo año, encuentran que cada integrante familiar, tiene una manera distinta de afrontar los sucesos vitales estresantes.

En la última pregunta del inventario de estrategias de afrontamiento relacionada con la percepción que tenía la paciente en relación a la capacidad que tiene para poder afrontar su situación actual, muestra claramente que esta percepción es fundamental para que lo lleve a cabo, por lo que la inteligencia emocional cobra gran importancia, ya que permite favorecer la resolución de problemas, redimensionar la enfermedad y disminuir el aislamiento en las pacientes con cáncer de mama, por lo que es relevante pensar en cursos o intervenciones que favorezcan la inteligencia emocional.

De acuerdo a lo encontrado en este trabajo y lo reportado en la literatura es de suma importancia indagar más sobre los aspectos que influyen en la paciente mediante una metodología cuantitativa y cualitativa que aporta estrategias para brindar una atención integral.

\section{CONCLUSIONES}

El sentimiento que con mayor frecuencia presentan las mujeres con cáncer de mama al recibir la noticia de su diagnóstico es el temor.

La resolución de problemas es la estrategia de afrontamiento que más utilizan las pacientes con esta afección.

La edad está estrechamente relacionada con los afrontamientos centrados en el problema y en las emociones.

A mayor escolaridad mayor reestructuración cognitiva, búsqueda de apoyo social y expresión emocional.

El tipo de tratamiento utilizado influye en la resolución de problemas de las pacientes con cáncer de mama de reciente diagnóstico. 
La estructura familiar y la percepción que tienen las pacientes de cáncer de mama influyen en su capacidad para afrontar dicho evento.

\section{AGRADECIMIENTOS}

A todas las pacientes que participaron en este estudio, así como el apoyo recibido por parte del personal de la Institución donde se llevó a cabo la aplicación del instrumento.

\section{REFERENCIAS BIBLIOGRÁFICAS}

1. McDonough $\mathrm{MH}$, Sabiston CM, Wrosch C. Predicting changes in posttraumatic growth and sugbetive well-being among breast cáncer survivors: The role of social support and stress. Psychooncology 2013. Doi: 10.1002/pon.3380

2. Robles R, Morales $M$, Jiménez LM, Morales J. Depresión y ansiedad en mujeres con cáncer de mama: el papel de la afectividad y el soporte social. Psicooncología. 2009;6:191-201.

3. Nigenda G, Lozano R, Arreola-Ornelas H, Langer A, Frenk J. Cáncer de mama en México: una prioridad apremiante. Salud Pública Mex 2009;51:335-44.

4. Cárdenas SJ, Sandoval GF. Tercera revisión del consenso nacional sobre diagnóstico y tratamiento del cáncer mamario.Ginecol Obstet Mex 2010;78:138-50.

5. Knaul FM, Arreola-Ornelas H, Velázquez E, Dorantes J, Méndez O, Ávila-Burgos L. El costo de la atención del cáncer mamario: el caso del Instituto Mexicano del Seguro Social. Salud Pública Mex 2009;51:286-95.

6. Bañuelos-Barrera $P$, Bañuelos-Barrera $Y$, Esquivel-Rodríguez M, Moreno-Ávila V. Autoconcepto de mujeres con cáncer de mama. Rev Enferm Inst Mex Seguro Soc 2007;15:129-34.

7. Hagedoorn M, Kreicbergs U, Appel C. Coping with cancer: The perspective of pa- tients' relatives. Acta Oncol 2011;50:20511.Doi: 10.3109/0284186X.2010.536165.

8. Knaul FM. Una mujer ante el cáncer de mama en México. Salud Pública Mex 2009;51:364-71.

9. Bellver A, Sánchez-Cánovas J, Santaballa A, Munárriz B, Pérez-Fidalgo JA, Montalar J. Mujeres con cáncer de mama: Evaluación del afecto positivo y negativo y valoración de un programa de intervención psicológica en el ámbito hospitalario. Psicooncología 2009;6:139-54.

10. Yusuf A, Hadi A, Mahamood Z, Keng SL. Understanding the breast cancer experience: A qualitative study of Malaysian women. Asian Pac J Cancer Prev 2013;14:3689-98.Doi: 10.7314/ APJCP.2013.14.6.3689

11. Narváez A, Rubiños C, Cortés-Funes F, Gómez R, García A. Valoración de la eficacia de una terapia grupal cognitivo-conductual en la imagen corporal, autoestima, sexualidad y malestar emocional (ansiedad y depresión) en pacientes de cáncer de mama. Psicooncología 2008;5: 93-102.

12. Olivares M, Martin M, Román J, Moreno A. Valoración psicosocial en pacientes con patología mamaria oncológica quirúrgica. Psicooncología 2010;7:81-97.

13. Khalili N, Farajzadegan Z, Mokanan F, Bahrami F. Coping strategies quality of life and pain in women with breast cancer. Iran J Nurs Midwifery Res 2013;18:105-11.

14. Bjorneklett HG, Rosenbland A, Lindemalm C, Ojutkangas ML, Letochalt N, Strang $\mathrm{P}$, et al. Long term follow-up of a randomized study of support group intervention in women with primary breast cancer. J Psychosom Res 2013;74:346-53. Doi: 10.1016/j.jpsychores.2012.11.005

15. Yoo W, Ghih MY, Kwon MW, Yang Y, Cho E, McLaughlin B, et al. Predictors of the change in the expression of emotional support within an online breast cancer support group: a longitudinal study. Patient Educ Couns 2013;90:88-95. Doi: 10.1016/j.pec.2012.10.001 
16. Millán-González R, Solano-Medina N. Duelo, duelo patológico y terapia interpersonal. Rev Colomb Psiquiatr 2010;39:375-88.

17. Díaz VE. Sexualidad, cuerpo y duelo: experiencia clínica con mujeres diagnosticadas con cáncer ginecológico o de mama. Pensamiento Psicológico 2010;7:155-60.

18. Herrera GP, Rodríguez Gl. Estudio piloto de validación del instrumento Brief Cope Inventory en español para evaluar estrategias de afrontamiento en pacientes con cáncer de seno. [Tesis de licenciatura]. Pontífica Universidad Javeriana. Facultad de Enfermería. Bogotá. 2009

19. Vargas SP. Confiabilidad del instrumento Brief Cope Inventory en español para evaluar estrategias de afrontamiento en pacientes con cáncer de seno. Tesis de licenciatura. Pontificia Universidad Javeriana. Facultad de Enfermería. Bogotá. 2009.

20. Font A, Cardoso A. Afrontamiento en cáncer de mama: pensamientos, conductas y reacciones emocionales. Psicooncología 2009;6: 27-42.

21. Mera P, Ortíz M. La relación del optimismo y las estrategias de afrontamiento con la calidad de vida de mujeres con cáncer de mama. Ter Psicol 2012;30:69-78. Doi: 10.4067/S0718-48082012000300007

22. Park B, Hwang S. Unmet needs breast cancer patients relative to survival duration. Yonsei Med J 2012;53:118-25. Doi:10.3349/ymj.2012.53.1.118

23. Mamani AE. Funcionamiento familiar $y$ afrontamiento el estrés en pacientes con cáncer de mama. [Tesis de licenciatura]. Universidad nacional Mayor de San Carlos. Universidad de Perú. Facultad de Psicología. 2008.

24. Berger AM, Lockhart K, Agrawal S. Variability of patterns of fatigue and quality of life over time based on different breast cancer adjuvant chemotherapy regimens. Oncol Nurs Forum 2009;35:563-70. Doi: 10.1188/09.ONF.563-570.

25. Varela B, Caporale B, Delgado L, Viera M, Galain A Y Schwartzmann L. Confronting the possibility of having breast cancer. Ciencia y Enfermería 2007 XIII: 33-40.

26. Fernández B, Bejar E, Campos M. Primer impacto: Programa de detección de distrés y atención psicosocial para paciente recién diagnosticado y sus familiares. Psicooncología 2012;9:317-34.Doi: 10.5209/ rev_PSIC.2013.v9.n2-3.40900

27. Hawkes A, Hughes K, Kutchitson S. Feasibility of brief psychological distress screening by a community-based telephone helpline for cancer patients and cares. BMJ Cancer 2010;10-4. Doi: 10.1186/1471-2407-10-14.

28. Cantu R, Alvarez J. Sucesos vitales y factores psicosociales asociados: el caso de pacientes con cáncer. Psicol Salud 2009; 19:21-32.

29. Cabrera A, Ferraz R. Impacto del cáncer en la dinámica familiar. Biomedicina 2011;6:42-8.

30. Stevina $M$, Nastran K. Family relationships and post-traumatic growth in breast cancer patients. Psychiatr Danub 2012;24:298306.

31. Cano FJ, Rodríguez L, Martínez J. adaptación del Inventario de Estrategias de Afrontamiento. Actas Esp Psiquiatr 2007;35:29-39.

32. Nava C, Ollua P, Vega C, Soria R. Inventario de Estrategias de Afrontamiento: una replicación. Psicol Salud 2010;20:213-20.

33. Varela B, Alonso R, Galain A, Ibañez A, Abreu M. Defensas y afrontamiento frente al cáncer de mama luego del diagnóstico y cirugía. Rev Psiquiatr Urug 2011;72:110-22.

34. Avis en, Levine B, Naughton MJ, Case LD, Naftalis E, Van Zee KJ. Age-related longitudinal changes in depressive symptoms following breast cancer diagnosis and treatment. Breast Cancer Res Treat 2013;139:199-206. Doi: 10.1007/s10549013-2513-2.

35. Díaz G, Yarigaño J. Clima familiar y afrontamiento al estrés en pacientes oncológicos. IIPSI. 2010;13:69-86. 
